\title{
Plasmid transfer in the animal intestine and other dynamic bacterial populations: the role of community structure and environment
}

\author{
Tine R. Licht, ${ }^{1,2}$ Bjarke B. Christensen, ${ }^{1}$ Karen A. Krogfelt ${ }^{2}$ \\ and Søren Molin ${ }^{1}$ \\ Author for correspondence: Søren Molin. Tel: +45 452525 13. Fax: +45 45887328. \\ e-mail:imsm@pop.dtu.dk
}

\footnotetext{
1 Department of Microbiology, Building 301, Technical University of Denmark, DK-2800 Lyngby, Denmark

2 Department of Gastrointestinal Infections, Statens Serum Institut, Artillerivej 5, DK-2300 Copenhagen S, Denmark
}

\begin{abstract}
The transfer of the R1drd19 plasmid between isogenic strains of Escherichia coli BJ4 in batch cultures of laboratory media and intestinal extracts was compared. Using an estimate of plasmid transfer rate that is independent of cell density, of donor: recipient ratios and of mating time, it was found that transfer occurs at a much lower rate in intestinal extracts than in laboratory media. Furthermore, the results suggest that the majority of intestinal plasmid transfer takes place in the viscous mucus layer covering the epithelial cells. Investigation of plasmid transfer in different flow systems harbouring a dynamic, continuously growing population of constant size showed that transfer kinetics were strongly influenced by bacterial biofilm formation. When donor and recipient populations were subjected to continuous mixing, as in a chemostat, transfer continued to occur at a constant rate. When donor and recipient populations retained fixed spatial locations, as in a biofilm, transfer occurred very rapidly in the initial phase, after which no further transfer was detected. From in vivo studies of plasmid transfer in the intestine of streptomycin-treated mice, results were obtained which were similar to those obtained in the biofilm, but differed markedly from those obtained in the chemostat. In spite of peristaltic movements in the gut, and of apparently even distribution of $E$. coli as single cells in the intestinal mucus, the intestinal environment displays transfer kinetics different from those expected of a mixed, liquid culture, but quite similar to those of a biofilm.
\end{abstract}

Keywords: plasmid, conjugation, chemostat, biofilm, intestine

\section{INTRODUCTION}

The importance of investigating plasmid transfer in natural environments has recently been emphasized by the increasing spread of multi-resistant bacterial pathogens (Davies, 1994), and the extensive use of antibiotic derivatives as growth promoters for domestic animals (Witte, 1998). In this context, transfer of genes encoding antibiotic resistance within the complex bacterial flora of the animal gut is of particular interest.

In spite of a large number of communications describing plasmid transfer in natural environments (Barkay et al., 1995 ; Kruse \& Sørum, 1994; Pukall et al., 1996; Rang et al., 1996; Top et al., 1990; Normander et al., 1998), there is a lack of studies which systematically compare plasmid transfer under different conditions and address more general questions about the mechanisms important for transfer processes. Many such questions can be answered by relatively simple experimental set-ups.

Previous attempts to predict the kinetics of plasmid transfer in the animal gut have been based on the assumption that the intestine can be compared to a chemostat (Freter et al., 1983a, c). One challenge to the chemostat model of the gut is that such a model implies that there is no compartmentalization of the intestinal environment. Escherichia coli cells grow rapidly in the intestinal mucus layer, whereas only very slow growth takes place in the gut contents (Wadolkowski et al., 1987; Poulsen et al., 1995; Licht et al., 1999). This suggests that plasmid transfer, which is believed to be inhibited by conditions of stress or starvation of the cells (Simonsen et al., 1990; Smets et al., 1993; Muela et al., 1994), mainly occurs within the fraction of intestinal $E$. coli present in the mucus (Poulsen et al., 1995). 
Experiments comparing plasmid transfer in intestinal mucus and contents are presented in this study.

Another challenge to the chemostat model concerns the assumption of perfect mixing of donor and recipient cells, which is also implicit in this type of gut model. To test whether this assumption is true for the intestinal population, we compared the intestine to other flow systems. The term 'flow system' denotes a system where a bacterial community of a limited size (i) continuously receives nutrients carried by a flow, and (ii) proliferates with a given mean rate that equals the mean rate by which bacterial cells are transported away from the system, i.e. the dilution rate. Examples of flow systems (or continuous cultures) are bacterial populations growing in chemostats, and bacterial biofilms formed on surfaces subjected to a flow of nutrients (Costerton et al., 1987; Woolfaardt et al., 1994). Intestinal mucus can be considered a flow system, since the E. coli population therein is continuously dividing with a mean doubling time of approximately $1 \mathrm{~h}$ (Poulsen et al., 1995), using the nutrients excreted from the epithelium as growth substrate, and shedding bacteria which are excreted with faeces. The population thus retains a constant size, even though the bacteria are continuously dividing.

Previous data indicated that $E$. coli colonizing the caecal and colonic mucus of streptomycin-treated mice are present only as single cells that are not attached to the epithelium (Poulsen et al., 1994). This suggests that mixing of the intestinal population could occur, and that the mucus, in spite of its high viscosity, may be considered as a liquid medium. Experiments presented in this study were designed to clarify whether plasmid transfer in the mouse gut displays the kinetics expected of a mixed liquid-flow system.

\section{METHODS}

Strains and laboratory media. A streptomycin-resistant derivative of Escherichia coli $\mathrm{BJ} 4$, which was isolated from a healthy Wistar rat at the National Food Agency, Denmark (Jacobsen et al., 1993), was used in all experiments. When acting as donor, this strain hosted R1drd19 (Freter et al., 1983b, Levin et al., 1979), which is a permanently transferderepressed mutant of the R1 plasmid provided by $\mathrm{K}$. Nordström, University of Uppsala, Sweden, and confers resistance to chloramphenicol and ampicillin. A derivative of E. coli $\mathrm{BJ} 4$, made resistant to rifampicin by $\mathrm{P} 1$ transduction, was used as recipient.

Bacteria were grown in the presence of $100 \mu \mathrm{g}$ streptomycin sulphate $\mathrm{ml}^{-1}$. In chemostat and batch culture experiments, AB minimal medium (Clark \& Maaløe, 1967) supplemented with $0 \cdot 2 \%$ glycerol was used. Additional batch culture experiments were carried out in L-broth (Bertani, 1951). In the biofilm experiments, $\mathrm{AB}$ minimal medium with only $0.02 \%$ glycerol was used. Selective platings were done on agar containing L-broth with $100 \mu \mathrm{g}$ streptomycin sulphate $\mathrm{ml}^{-1}$ in addition to either (i) $20 \mu \mathrm{g}$ chloramphenicol ml $\mathrm{m}^{-1}$, selecting for donors and transconjugants, (ii) $100 \mu \mathrm{g}$ rifampicin $\mathrm{ml}^{-1}$, selecting for recipients and transconjugants, or (iii) $20 \mu \mathrm{g}$ chloramphenicol $\mathrm{ml}^{-1}+100 \mu \mathrm{g}$ rifampicin $\mathrm{ml}^{-1}$, selecting for transconjugants. Antibiotics were purchased from Sigma.

Preparation of intestinal extracts for in vitro incubation.
Intestinal extracts were prepared as previously described (Poulsen et al., 1995; Licht et al., 1999). Six- to eight-week-old outbred albino female SSc: CF1 mice (Statens Serum Institut, Copenhagen, Denmark) were treated for $24 \mathrm{~h}$ with $5 \mathrm{~g}$ streptomycin sulphate $1^{-1}$ in drinking water. The mice were then killed by cervical dislocation, and their caeca were removed and carefully cut open with a scalpel. The part of the caecal contents that was released without manipulation was transferred to Eppendorf tubes and kept on ice for no longer than $2 \mathrm{~h}$ until use. Care was taken that no mucus was mixed with the contents. The caeca were first washed in $5 \mathrm{ml}$ cold phosphate-buffered saline (Statens Serum Institut, Copenhagen, Denmark), then the mucus was scraped from the caecal epithelial cells using a rubber spatula. The mucus was kept on ice for a maximum of $2 \mathrm{~h}$ until use.

Batch culture experiments. Equal amounts of donor and recipient bacteria were inoculated into either L-broth, AB medium with glycerol, mouse caecal contents or mouse caecal mucus to a concentration of approximately $10^{4}$ c.f.u. $\mathrm{ml}^{-1}$. A number of samples were taken from the batch cultures during exponential growth, and a few additional samples were taken after entry into stationary phase. The samples were diluted in cold $0.9 \%(\mathrm{w} / \mathrm{v}) \mathrm{NaCl}$ and plated on selective agar as described above. The batch culture matings in laboratory media were performed twice, while the mating experiments in intestinal extracts were performed four times.

The specific growth rates $\mu\left(\mathrm{h}^{-1}\right)$ in different media were calculated from samples taken during exponential growth phase, $\mu=\ln \left(N_{2} / N_{1}\right) /\left(t_{2}-t_{1}\right)$, where $N_{1}$ and $N_{2}$ designate the total population densities obtained at times $t_{1}$ and $t_{2}$. The plasmid transfer rate parameter $\gamma\left(\mathrm{ml} \mathrm{cell}^{-1} \mathrm{~h}^{-1}\right)$ in each substrate was calculated from samples taken during stationary phase using the end-point method published by Simonsen et al. $(1990): \gamma=\mu \ln (1+T N / R D) /\left(N-N_{0}\right)$, where $R, D$ and $T$ denote the densities of recipients, donors and transconjugants, respectively, and $N_{0}$ and $N$ are the total population densities at the time of inoculation and at the time of sampling, respectively. This estimate of plasmid transfer rate is independent of cell density, donor : recipient ratio and mating time. The standard deviation of the $\gamma$ estimates obtained varied between $92 \%$ and $103 \%$, which is in agreement with previous observations (Simonsen et al., 1990). The transfer rates measured in batch cultures were compared using Student's $t$ test.

Chemostat experiments. Six chemostats with a volume of approximately $45 \mathrm{ml}$ were established. For mixing and aeration, air bubbles were continuously pumped through the medium. The flow rate of the substrate was $11.4 \mathrm{ml} \mathrm{h}^{-1}$, resulting in a dilution rate $v=11 \cdot 4 / 45=0 \cdot 25 \mathrm{~h}^{-1}$. Bacteria were grown overnight in glycerol-supplemented $\mathrm{AB}$ medium prior to inoculation. The recipient strain was inoculated $\left(10^{9}\right.$ c.f.u. $)$, and allowed to establish a stable population size before the donor was introduced $5 \mathrm{~d}$ later. In three chemostats, high numbers of donors $\left(10^{9}\right.$ c.f.u. $)$, and in three others, lower numbers of donors $\left(10^{6}\right.$ c.f.u.) were introduced. Six hours after donor inoculation an effluent sample was taken. During the following $8 \mathrm{~d}$, samples were taken, diluted and plated on selective media. The experiment was performed twice.

Biofilm experiments. Bacteria were grown overnight in glycerol-supplemented $\mathrm{AB}$ medium prior to inoculation. Biofilms inoculated with $6 \times 10^{8}$ c.f.u. of the recipient strain were cultivated in six rectangular flow channels (Wolfaardt et al., 1994) with individual dimensions of $1 \times 4 \times 40 \mathrm{~mm}$. Equipment, maintenance and sterilization of the system was as previously described (Christensen et al., 1998; Møller et al., 1998). The dilution rate of the medium was $v=18 \mathrm{~h}^{-1}$. Stable 
biofilms consisting of recipients only were allowed to establish for 1 week, after which the donor was introduced at high numbers $\left(6 \times 10^{8}\right.$ c.f.u. $)$ in three channels, and at low numbers $\left(6 \times 10^{4}\right.$ c.f.u. $)$ in the other three. An effluent sample was taken $5 \mathrm{~h}$ after inoculation of the donors. During the next $8 \mathrm{~d}$, samples were taken, diluted and plated on selective media. The experiment was performed twice.

Colonization of streptomycin-treated mice. Six mice (as specified above) were given sterile water containing $5 \mathrm{~g}$ streptomycin sulphate $\mathrm{l}^{-1}$. After $24 \mathrm{~h}$, faecal samples were taken, homogenized in $0.9 \%(\mathrm{w} / \mathrm{v}) \mathrm{NaCl}$ and plated on $\mathrm{LB}$ with streptomycin as negative controls. Bacteria were grown overnight in L-broth prior to inoculation. A suspension of the E. coli BJ4 recipient strain $\left(5 \times 10^{9}\right.$ c.f.u.) in $100 \mu \mathrm{l} 20 \%$ (w/v) sucrose was given to the mice per os. The recipient was allowed to establish in the intestine for 1 week, before the donor was given to the mice per os. Three mice received $9 \times 10^{8}$ c.f.u. of the donor, whereas the other three received $9 \times 10^{4}$ c.f.u. The experiment proceeded for $7 \mathrm{~d}$ after inoculation of the donor strain. The mice were individually caged, the cages being changed daily, and the mice continuously receiving drinking water containing streptomycin. The numbers of c.f.u. of donors, recipients and transconjugants per $\mathrm{g}$ faeces were determined by dilution in $0.9 \%$ $\mathrm{NaCl}$ and spreading on selective LB plates as specified above. The experiment was performed three times.

Theory for plasmid transfer in chemostats. Levin et al. (1979) proposed a mass-action model for plasmid transfer in a liquid batch culture based on the assumptions that (i) mating occurs randomly with a frequency that is jointly proportional to the donor and recipient populations, and independent of the bacterial growth rate, (ii) segregation is negligible, (iii) transconjugants can transmit the plasmid as soon as they have received it, (iv) the plasmid transfer frequencies of donors and transconjugants are similar, and (v) growth rates of donors, recipients and transconjugants are similar. Letting $\mu$ represent the rate of bacterial growth and $\gamma$ the conjugative transfer rate constant, the differentiations with respect to time of the donor $(D)$, recipient $(R)$ and transconjugant $(T)$ densities were then given by (Levin et al., 1979):

$\mathrm{d} R / \mathrm{d} t=\mu R-\gamma(D+T) R$

$\mathrm{d} D / \mathrm{d} t=\mu \mathrm{D}$

$\mathrm{d} T / \mathrm{d} t=\mu T+\gamma(D+T) R$

Note that a consequence of assumption (i) mentioned above is that the donor, recipient and transconjugant populations described by the three equations must be subjected to continuous mixing.

In a flow system, where the bacteria are removed from the population with the dilution rate $v$, the above equations can be modified as follows:

$\mathrm{d} R / \mathrm{d} t=\mu \mathrm{R}-\gamma(D+\mathrm{T}) R-v R$

$\mathrm{d} D / \mathrm{d} t=\mu \mathrm{D}-v D$

$\mathrm{d} T / \mathrm{d} t=\mu \mathrm{T}+\gamma(D+T) R-v T$

When a steady state is reached, the total bacterial density in the system $(R+D+T)$ remains constant, and the growth rate $\mu$ equals the dilution rate $v$, thus:

$\mathrm{d} R / \mathrm{d} t=-\gamma(D+T) R$

$\mathrm{d} D / \mathrm{d} t=0$

$\mathrm{d} T / \mathrm{d} t=\gamma(D+T) R$

In other words, this means that in a steady-state chemostat where perfect mixing of the donor, recipient and trans- conjugant populations occurs, the donor population will remain constant, while the recipient population will decrease with a rate which equals the rate of increase of the transconjugant population. When the recipient population has disappeared $(R=0)$, none of the population densities will change further.

Since $D$ is constant, and also the total population density $N=D+T+R$ is constant, equation (3b) can be solved. When transconjugants $T=0$ at time $t=0$, we get

$T=[(N-D)(\exp (N \gamma t)-1)] /[(N / D)+\exp (N \gamma t)-1]$

The number of transconjugants present in the chemostat expressed as a function of time is therefore predicted to follow the equation given above. $T$ is approaching $N-D$ when time increases.

When $T \ll D$, equation (3b) can be reduced to:

$\mathrm{d} T / \mathrm{d} t=\gamma \mathrm{DR}$

while, when $\mathrm{D} \ll \mathrm{T}$, the similar reduction gives

$\mathrm{d} T / \mathrm{d} t=\gamma T R$

As long as the recipient population is much larger than the population of plasmid-carrying strains $[(D+T) \ll R]$, the relative change in the recipient population will be negligible, and the solutions to equations (3d) and (3e) can then, respectively, be approached by:

$T=\gamma D R t(T \ll D \ll R)$

and

$T=T_{1} \exp \left[\gamma R\left(t-t_{1}\right)\right](D \ll T \ll R)$

where $T_{1}$ is the number of transconjugants present at the time $t_{1}$ where the kinetics start following equation $(3 \mathrm{~g})$.

The increase in the transconjugant population expressed as a function of time thus initially approaches linearity (equation $3 \mathrm{f})$, thereafter an exponential function (3g), and finally becomes constant when $R=0$.

\section{RESULTS}

\section{Comparison of transfer rate constants in batch cultures}

Low numbers of E. coli BJ4 Rif ${ }^{\mathrm{R}}$ (recipient) and E. coli $\mathrm{BJ} 4 / \mathrm{R} 1 d r d 19$ (donor) were inoculated together into four different media supporting very different growth conditions: (i) L-broth, (ii) glycerol-supplemented minimal medium, (iii) caecal mucus isolated from streptomycin-treated mice, and (iv) caecal contents isolated from streptomycin-treated mice. Numbers of donors, recipients and transconjugants were followed by plating on selective agar plates at several different time points. From these plate counts, the growth rates of the batch cultures during exponential growth were determined, and the transfer rate constants for the four different media were calculated using the end-point method published by Simonsen et al. (1990). The equation applied is given in Methods. The estimates of the rates of transfer obtained in this fashion are absolute and independent of the kinetics of the mating process (Simonsen et al., 1990). The growth rates $(\mu)$ and transfer 
Table 1. Growth rates $(\mu)$ and transfer rate parameters $(\gamma)$ measured by the end-point method

See Simonsen et al. (1990) for details of the end-point method. Generation times $\left(\ln 2 \mu^{-1}\right)$ are included for clarity. Measurements were done in batch cultures of (i) L-broth, (ii) AB minimal medium supplemented with $0 \cdot 2 \%$ glycerol, (iii) caecal mucus from streptomycin-treated mice, and (iv) caecal contents from streptomycin-treated mice.

\begin{tabular}{|lcccc|}
\hline & LB & AB + glycerol & Mucus & Contents \\
\hline Growth rate, $\mu\left(\mathrm{h}^{-1}\right)$ & $1 \cdot 61 \pm 0 \cdot 27$ & $0 \cdot 54 \pm 0 \cdot 02$ & $1 \cdot 14 \pm 0 \cdot 24$ & $0 \cdot 32 \pm 0 \cdot 09$ \\
Generation time $(\mathrm{min})$ & $26 \cdot 5$ & $77 \cdot 4$ & $37 \cdot 3$ & $134 \cdot 0$ \\
Transfer rate, $\gamma\left(\mathrm{ml} \mathrm{cell}{ }^{-1} \mathrm{~h}^{-1}\right)$ & $2 \cdot 52 \times 10^{-11}$ & $2 \cdot 99 \times 10^{-11}$ & $6 \cdot 99 \times 10^{-12}$ & $5 \cdot 33 \times 10^{-13}$ \\
SD of $\gamma$ values & $\pm 1 \cdot 97 \times 10^{-11}$ & $\pm 3 \cdot 19 \times 10^{-11}$ & $\pm 7 \cdot 18 \times 10^{-12}$ & $\pm 4.93 \times 10^{-13}$ \\
\hline
\end{tabular}

(a)

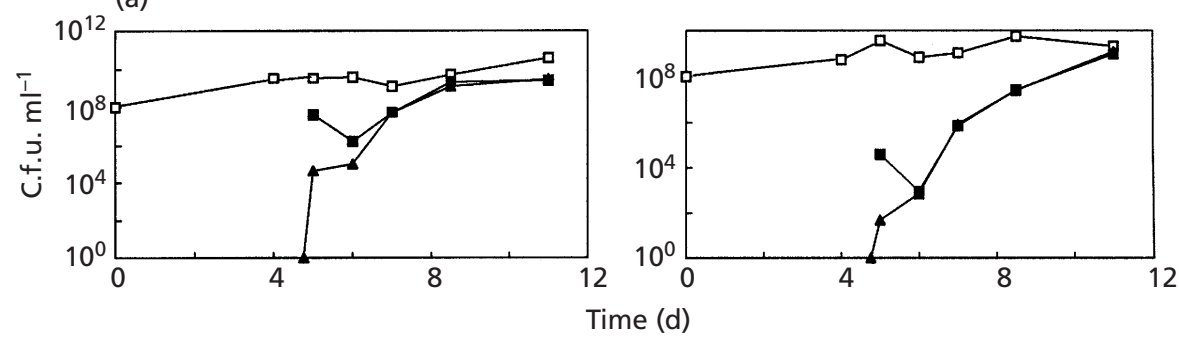

(b)

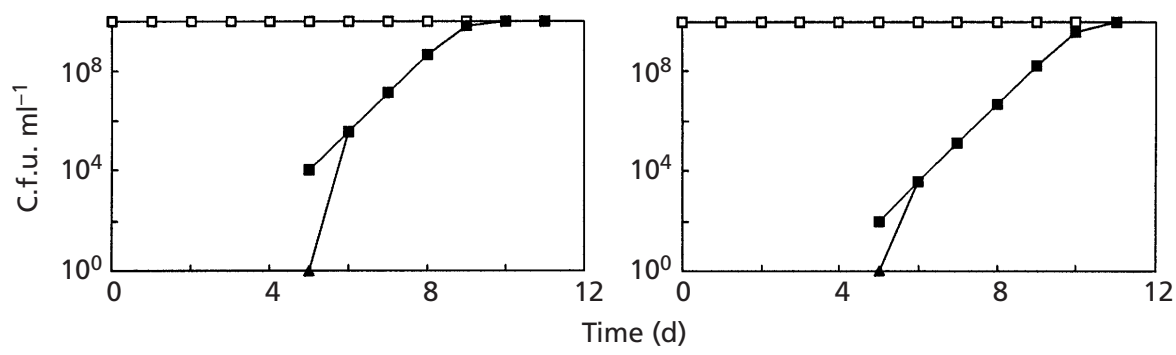

Fig. 1. (a) Chemostat mating experiments in $A B$ minimal medium supplemented with glycerol. Recipients were introduced at day 0 . At day 5, either $10^{9}$ (left) or $10^{6}$ (right) donor cells were introduced $6 \mathrm{~h}$ before sampling of the effluent. Recipients + transconjugants $(\square)$, donors + transconjugants $(\boldsymbol{\square})$ and transconjugants $(\boldsymbol{\Delta})$ were quantified by plating of effluent samples on selective plates. The numbers of transconjugants at the time of donor introduction were set to 1. For clarity, representative data from two chemostats are selected. (b) Mathematical prediction of transfer kinetics in a chemostat, where the bacteria are subjected to perfect mixing. Numbers of recipients + transconjugants ( $\square$ ), donors + transconjugants $(\boldsymbol{\square})$ and transconjugants $(\boldsymbol{\Delta})$ were calculated using equation (3c) from Methods. The numbers of transconjugants at the time of donor introduction were set to 1 . An initial recipient concentration of $10^{10} \mathrm{c} . \mathrm{f} . \mathrm{u}$. $\mathrm{ml}^{-1}$, and initial donor concentrations of either $10^{4}$ c.f.u. $\mathrm{ml}^{-1}$ (left) or $10^{2}$ c.f.u. $\mathrm{ml}^{-1}$ (right), were chosen in order to match the concentrations measured after stabilization in the experimental system. A transfer rate constant $(\gamma)$ of $1.5 \times 10^{-11}$ was used, in agreement with the experimental estimates given in Table 1.

rate constants $(\gamma)$ obtained are given in Table 1. Statistical analysis of the mean $\gamma$ values presented revealed that the transfer rate constants obtained in Lbroth and glycerol-supplemented minimal media were not different from each other. However, the transfer rate constants obtained during in vitro growth in caecal mucus and caecal contents were 4- and 50-fold lower, respectively, than observed in laboratory media $(0.05<P<0.1$ and $0.02<P<0.05)$. Furthermore, the $\gamma$ values obtained in caecal mucus and contents were significantly different from each other $(0 \cdot 05<P<0 \cdot 1)$, the transfer rate in contents being 13 -fold lower than in mucus.

\section{Transfer in chemostats}

Six $45 \mathrm{ml}$ chemostats carrying a flow of minimal medium supplied with glycerol were inoculated with the recipient, E. coli $\mathrm{BJ} 4 \mathrm{R}^{\mathrm{R}}{ }^{\mathrm{R}}$. Five days later, when the size of the recipient population was stable, E. coli BJ4/ $\mathrm{R} 1 d r d 19$ (donor) was introduced at high numbers to three of the chemostats, and at $10^{3}$-fold lower numbers to the three others.

The numbers of (i) recipients + transconjugants, (ii) donors + transconjugants, and (iii) transconjugants in the effluent were determined by plating on selective agar plates (Fig. 1a). The experiment proceeded over a period 


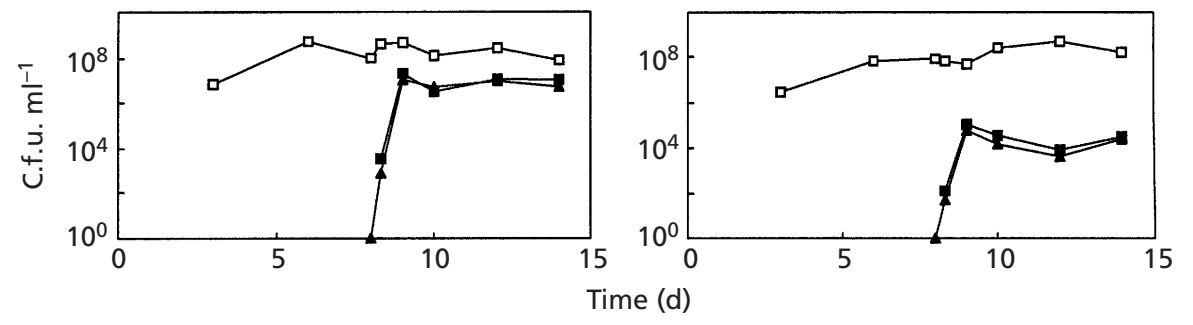

Fig. 2. Biofilm mating experiments in $A B$ minimal medium supplemented with glycerol. Recipients were introduced at day 0 . At day 8 , either $2 \times 10^{8}$ (left) or $2 \times 10^{4}$ (right) donor cells were introduced $5 \mathrm{~h}$ before sampling of the effluent. Recipients + transconjugants $(\square)$, donors + transconjugants $(\boldsymbol{\square})$ and transconjugants $(\boldsymbol{\Delta})$ were quantified by plating of effluent samples on selective plates. The numbers of transconjugants at the time of donor introduction were set to 1 . For clarity, representative data from two flow channels are selected.

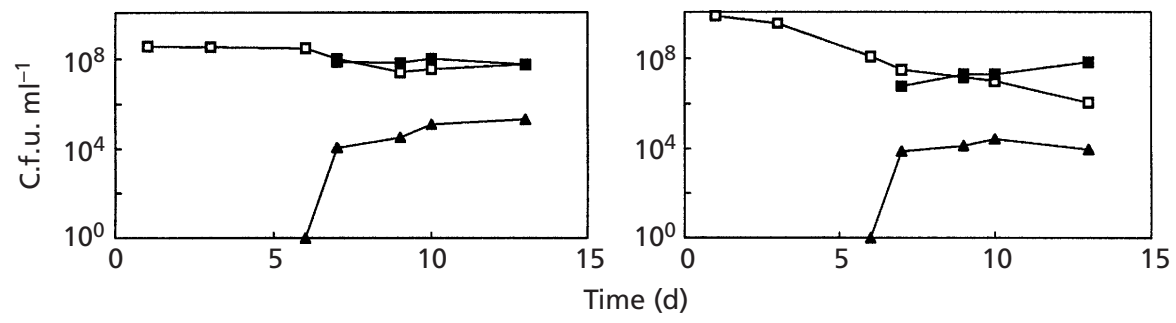

Fig. 3. Mating experiments in the mouse intestine. The recipient strain was given to the mice per os at day 0 . At day 6 , the mice received $9 \times 10^{8}$ donor cells. Recipients + transconjugants $(\square)$, donors + transconjugants $(\square)$ and transconjugants (A) were quantified by plating of faecal samples on selective plates. The numbers of transconjugants at the time of donor introduction were set to 1 . For clarity, representative data from two mice are selected.

of $8 \mathrm{~d}$. We observed a continuous increase in the number of transconjugants, which eventually replaced recipient cells in the population. Fig. 1(b) shows the predicted development of the same three populations, assuming that the numbers of transconjugants followed equation (3c) given in Methods, using a transfer rate parameter $(\gamma)$ calculated above in one of the laboratory media batch cultures. From Fig. 1 it is clear that except for a short period of imbalance in the chemostat following donor inoculation, the transfer kinetics in the chemostat followed the predictive calculations.

\section{Transfer in biofilms}

Six flow channels carrying a flow of minimal medium supplemented with glycerol were inoculated with the recipient strain, E. coli BJ4 Rif ${ }^{\mathrm{R}}$. Eight days later, this strain had formed a biofilm of a stable size, and high numbers of the donor strain (E. coli BJ4/R1drd19) were introduced into three channels, while $10^{4}$-fold lower numbers of donor cells were introduced into the remaining three channels. The numbers of (i) recipients + transconjugants, (ii) donors + transconjugants, and (iii) transconjugants in the effluent were determined by plating on selective agar plates (Fig. 2). The experiment proceeded for $8 \mathrm{~d}$ after introduction of the donors. The transfer kinetics were very different from what was observed in the chemostat. Initially, i.e. the first day, transfer occurred at a very high rate. Thereafter, no further transfer was observed, even though a large recipient population still persisted. At the end of the experiments, the large majority of the plasmid-carrying cells were transconjugants.

\section{Transfer in the mouse intestine}

To obtain intestinal E. coli populations large enough to result in detectable plasmid transfer, we used the streptomycin-treated mouse model (Que et al., 1986), which allows E. coli $\mathrm{BJ} 4$ to colonize the mouse large intestine at levels between $10^{8}$ and $10^{9}$ c.f.u. per $\mathrm{g}$ faecal material (Poulsen et al., 1995).

Six mice were inoculated per os with E. coli BJ4 Rif ${ }^{\mathrm{R}}$ (recipient). After 1 week, the strain had established in the mouse intestine, and high numbers of E. coli $\mathrm{BJ} 4 / \mathrm{R} 1 d r d 19$ (donor) were given to three of the mice, while $10^{4}$-fold lower numbers were given to the rest. The numbers of (i) recipients + transconjugants, (ii) donors + transconjugants, and (iii) transconjugants present in faecal samples were determined by plating on selective agar plates. For the mice receiving low numbers of donors, the size of the inoculum was not sufficient for the donor strain to establish itself in the intestine, and consequently no detectable transfer occurred (data not shown). For the other three mice, the donor was able to establish, and transfer occurred at a high rate initially after introduction of the donors (Fig. 3). Thereafter, no detectable transfer occurred. The recipient strain 
persisted at high numbers throughout the experiment, which continued for $7 \mathrm{~d}$ after introduction of the donor strain. The dominating fraction of the plasmid-carrying strains continued to be the original donors.

\section{DISCUSSION}

Bacterial growth in the animal intestinal environment has been suggested to resemble that in continuous-flow cultures or chemostats (Freter et al., 1983a, b, c). We have subjected two of the assumptions implicit in such chemostat-models of the intestine to further study: (i) that the animal gut is a non-compartmentalized environment for plasmid transfer, and (ii) that the intestinal bacterial population is subject to perfect mixing.

Kinetic coefficients determined for batch-grown cells can be predictive of their behaviour under continuousgrowth conditions, such as those in the intestinal environment (Smets et al., 1993). Therefore, we compared batch-culture transfer kinetics in extracts from different intestinal compartments to those observed in defined laboratory media, and to each other. One explanation for the lower transfer rate constants measured during in vitro exponential growth of bacteria in non-sterile caecal mucus compared to those measured in laboratory media (Table 1) could be that the higher viscosity of intestinal mucus may prevent efficient mixing of donor and recipient cells, and reduce motility of the E. coli cells (McCormick et al., 1990). Another possible explanation is that specific compounds in mucus might repress transfer efficiency, although to our knowledge, no report of such compounds currently exists. Transfer rate constants measured after inoculation in caecal content (which is less viscous than mucus) were about 13 -fold lower than observed in mucus (Table 1). In agreement with data obtained previously (Wadolkowski et al., 1988; McCormick et al., 1988; Poulsen et al., 1995; Licht et al., 1999), the bacterial growth rate in caecal contents was also significantly lower than in mucus (generation times of 134 and $37 \mathrm{~min}$, respectively), but a reduced growth rate per se should not result in lower transfer, as shown for the slow-growing batch culture in glycerol-supplemented minimal medium (Table 1). The presence of a compound in intestinal contents that inhibits bacterial growth by disturbing the processing of rRNA transcripts (Licht et al., 1999) might interfere with the process of plasmid transfer. The data presented in Table 1 strongly suggest that the viscous mucus layer covering the apical surface of epithelial cells is the intestinal compartment where the large majority of plasmid transfer takes place.

To address the question of whether donors and recipients in the intestine are subject to continuous mixing, we compared the transfer kinetics of the mouse intestine to those of two other flow systems: the chemostat and the biofilm. In all cases investigated, transfer from an incoming donor strain to an established recipient population was investigated.

The chemostat experiments showed that under conditions of perfect mixing of donor, recipient and transconjugant populations, the number of transconjugants increased with time until all recipients had received a plasmid (Fig. 1a). The kinetics of this process was in agreement with predictive calculations based on the theory for chemostats (Fig. 1b). Soon after donor introduction, when the number of donor cells was much higher than the number of transconjugants $(T \ll D)$, the increase in transconjugant cells was very rapid. Subsequently, when the number of plasmid-carrying bacteria was much lower than the number of recipient cells $(T+D \ll R)$, the increase in the transconjugant population occurred as an exponential function of time, until all recipients had become transconjugants. These kinetics were predicted from equations $(3 \mathrm{f})$ and $(3 \mathrm{~g})$ in Methods.

In the biofilm experiments, bacteria are allowed to attach to and colonize the glass surface of a flow chamber, instead of being continuously mixed. As a consequence of this, the local density of bacteria in the biofilm is higher than in the chemostat. Each bacterial cell will stay in a fixed position in the time between attachment and detachment from the biofilm. Plasmid transfer occurred within the biofilm at a high rate initially after introduction of the donor, after which no further transfer was observed (Fig. 2). The fixed position of the resident recipient cells and the high cell density probably facilitated initial formation of mating pairs, when donor cells attached. However, when all initial recipients had received a plasmid, further transfer was severely reduced, since the donor bacteria were also located in fixed positions. In contrast to what was observed in the chemostat, the recipient cells continued to constitute a large fraction of the biofilm population, indicating that the plasmid was not passed on from primary transconjugants to neighbouring recipient cells. In a previous study of plasmid transfer in biofilms (Christensen et al., 1998), in which the transconjugants were visualized by in situ techniques, it was shown that the transconjugants form a 'layer' with a thickness of a few bacteria, which remains on top of the recipient micro-colonies attached to the glass surface without transferring the plasmid to the lower layers of recipients. Although the plasmid used in that study is different from $\mathrm{R} 1 d r d 19$, the observations of the spatial position of transconjugants might be generally valid for plasmid transfer in biofilms. It is possible that at least one cell division is required before a newly formed transconjugant can transfer its acquired plasmid to another bacterium, as has been observed for Gram-positive species (Andrup et al., 1998). In the lower parts of biofilms there may be conditions of reduced nutrient availability (Costerton et al., 1994), resulting in very low rates of cell division.

The observed kinetics of plasmid transfer in the mouse intestine showed many similarities to the transfer kinetics of a biofilm: after a brief period with rapid transfer, no further increase in the number of transconjugants was observed even though the E. coli population of intestinal mucus is rapidly dividing (Wadolkowski et al., 1988; Poulsen et al., 1995; Licht et 
al., 1999), and should consequently be in a situation allowing continued plasmid transfer (Fig. 3). This suggests that bacteria populating the mucus layer, which is the compartment where the majority of plasmid transfer takes place (Table 1), retain fixed spatial positions. The peristaltic movements of the gut, and the fact that the mucus is a 'fluid' in spite of its high viscosity, is thus not enough to cause efficient mixing of donor and recipient populations, which would result in 'chemostat' transfer kinetics.

As the E. coli population constitutes only about $1 \%$ of all bacteria in the intestine of streptomycin-treated mice (Drasar \& Barrow, 1985), only few potential recipient cells will be in contact with incoming donor cells. When these recipients have received the plasmid, they will not be able to transfer it further unless the mucus population is mixed. The viscous nature of the intestinal mucus may support the initial formation of mating pairs between bacteria, but bacterial movement and subsequent formation of new mating pairs between displaced bacterial cells may on the other hand be severely restricted. This suggests that plasmid transfer from incoming donor cells occurs only immediately after establishment in the intestine. It also explains why a higher number of introduced donor cells resulted in detectable levels of transconjugants (Fig. 3), while a low dose of donors did not (data not shown).

The observations reported here are important to keep in mind when using data obtained in chemostat experiments to predict and interpret plasmid transfer events taking place in the intestinal environment. Furthermore, the results presented provide information relevant to events following ingestion of food contaminants carrying plasmids. We have shown that rapid transfer occurs immediately after introduction of the donor. Therefore, the transient passing of cells carrying conjugative plasmids represents a potential source of spread of resistance genes and other plasmid-encoded features to the indigenous intestinal flora.

\section{ACKNOWLEDGEMENTS}

We thank Anne Nielsen, Department of Microbiology, Technical University of Denmark, for technical assistance, and K. Nordström, University of Uppsala, Sweden, for providing the plasmid used. This work was financed by a grant from the Danish Medical Research Council to K.A.K. and S.M., a grant from the Danish Ministries of Health, Food Agriculture and Fisheries (DANMAP) to Statens Serum Institute, and by grants from the Danish Biotechnology Program to S.M. We dedicate this article to the memory of our late colleague, Lars Boe, whose lines of thought have been an inspiration to the present work.

\section{REFERENCES}

Andrup, L., Smidt, L., Andersen, K. \& Boe, L. (1998). Kinetics of conjugative transfer: a study of the plasmid pXO16 from Bacillus thuringiensis supsp. israelensis. Plasmid 40, 30-43.

Barkay, T., Kroer, N., Rasmussen, L. D. \& Sørensen, S. J. (1995).
Conjugal transfer at natural population densities in a microcosm simulating an estuarine environment. FEMS Microbiol Ecol 16, 43-54.

Bertani, G. (1951). Studies on lysogenesis. I. The mode of phage liberation by lysogenic Escherichia coli. J Bacteriol 62, 293-300.

Christensen, B. B., Sternberg, C., Andersen, J. B., Eberl, L., Møller, S., Givskov, M. \& Molin, S. (1998). Establishment of new genetic traits in a microbial biofilm community. Appl Environ Microbiol 64, 2247-2255.

Clark, D. J. \& Maaløe, O. (1967). DNA replication and the division cycle in E. coli. J Mol Biol 23, 99-112.

Costerton, J. W., Cheng K. J., Geesey, G. G., Ladd, T. I., Nickel, J. C., Dasgupta, M. \& Marrie, J. T. (1987). Bacterial biofilms in nature and disease. Annu Rev Microbiol 42, 435-464.

Costerton, J. W., Lewandowski, Z., DeBeer, D., Caldwell, D., Korber, D. \& James, G. (1994). Minireview: Biofilms, the customized microniche. J Bacteriol 176, 2137-2142.

Davies, J. (1994). Inactivation of antibiotics and the dissemination of resistance genes. Science 264, 375-382.

Drasar, B. S. \& Barrow, P. A. (1985). Intestinal Microbiology. Washington, DC: American Society for Microbiology.

Freter, R., Brickner, H., Botney, M., Cleven, D. \& Aranki, A. (1983a). Mechanisms that control bacterial populations in continuous flow-culture models of mouse large intestinal flora. Infect Immun 39, 676-685.

Freter, R., Freter, R. R. \& Brickner, H. (1983b). Experimental and mathematical models of Escherichia coli plasmid transfer in vitro and in vivo. Infect Immun 39, 60-84.

Freter, R., Stauffer, E., Cleven, D., Holdeman, L. V. \& Moore, W. E. C. (1983c). Continuous flow cultures as in vitro models of the ecology of large intestinal flora. Infect Immun 39, 666-675.

Jacobsen, B., Schlundt, J. \& Fisher, G. (1993). Study of a conditional suicide system for biological containment of bacteria in germ-free rats. Microb Ecol Health Dis 6, 109-118.

Kruse, H. \& Sørum, H. (1994). Transfer of multiple drug resistance plasmids between bacteria of diverse origins in natural microenvironments. Appl Environ Microbiol 60, 4015-4021.

Levin, B. R., Stewart, F. M. \& Rice, V. A. (1979). The kinetics of conjugative plasmid transmission: fit of a simple mass action model. Plasmid 2, 247-260.

Licht, T. R., Tolker-Nielsen, T., Holmstrøm, K., Krogfelt, K. A. \& Molin, S. (1999). Inhibition of Escherichia coli precursor-16S rRNA processing by mouse intestinal contents. Environ Microbiol 1, 23-32.

McCormick, B. A., Stocker, B. A. D., Laux, D. C. \& Cohen, P. S. (1988). Roles of motility, chemotaxis, and penetration through and growth in intestinal mucus and the ability of an avirulent strain of Salmonella typhimurium to colonize the large intestine of streptomycin-treated mice. Infect Immun 56, 2209-2217.

McCormick, B. A., Laux, D. C. \& Cohen, P. S. (1990). Neither motility nor chemotaxis plays a role in the ability of Escherichia coli F-18 to colonize the streptomycin-treated mouse large intestine. Infect Immun 58, 2957-2961.

Møller, S., Sternberg, C., Andersen, J. B., Christensen, B. B. \& Molin, S. (1998). In situ gene expression in mixed culture biofilms: evidence of interactions between community members. Appl Environ Microbiol 64, 721-732.

Muela, A., Pocino, M., Arana, I., Josto, J. I., Iriberri, J. \& Barcina, I. (1994). Effect of growth phase and parental cell survival in river water on plasmid transfer between Escherichia coli strains. Appl Environ Microbiol 60, 4273-4278. 
Normander, B., Christensen, B. B., Molin, S. \& Kroer, N. (1998). Effect of bacterial distribution and activity of on conjugal gene transfer on the phylloplane of the bush bean (Phaseolus vulgaris). Appl Environ Microbiol 64, 1902-1909.

Poulsen L. K., Lan, F., Kristensen, C. S., Hobolth, P., Molin, S. \& Krogfelt, K. A. (1994). Spatial distribution of Escherichia coli in the mouse large intestine inferred from rRNA in situ hybridization. Infect Immun 62, 5191-5194.

Poulsen, L. K., Licht, T. R., Rang, C., Krogfelt, K. A. \& Molin, S. (1995). Physiological state of Escherichia coli BJ4 growing in the large intestines of streptomycin-treated mice. J Bacteriol 177, 5840-5845.

Pukall, R., Tschäpe, H. \& Smalla, K. (1996). Monitoring the spread of broad host and narrow host range plasmids in soil microcosms. FEMS Microbiol Ecol 20, 53-66.

Que, J. U., Casey, S. W. \& Hentges, D. J. (1986). Factors responsible for increased susceptibility of mice to intestinal colonization after treatment with streptomycin. Infect Immun 53, 116-123.

Rang, C. U., Kennan, R. M., Midtvedt, T., Chao, L. \& Conway, P. L. (1996). Transfer of the plasmid RP1 in vivo in germ free mice and in vitro in gut extracts and laboratory media. FEMS Microbiol Ecol 19, 133-140.
Simonsen, L., Gordon, D. M., Stewart, F. M. \& Levin, B. R. (1990). Estimating the rate of plasmid transfer: an end-point method. J Gen Microbiol 136, 2319-2325.

Smets, B. F., Rittman, B. E. \& Stahl, D. A. (1993). The specific growth rate of Pseudomonas putida PAW1 influences the conjugal transfer rate of the TOL plasmid. Appl Environ Microbiol 59, 3430-3437.

Top, E., Mergeay, M., Springael, D. \& Verstraete, W. (1990). Gene escape model: transfer of heavy metal resistance genes from Escherichis coli to Alcaligenes eutrophus on agar plates and in soil samples. Appl Environ Microbiol 56, 2471-2479.

Wadolkowski, E. A., Laux, D. C. \& Cohen, P. S. (1988). Colonization of the streptomycin treated mouse large intestine by a human fecal Escherichia coli strain: role of growth in mucus. Infect Immun 56, 1030-1035.

Witte, W. (1998). Medical consequences of antibiotic use in agriculture. Science 279, 996-997.

Wolfaardt, G. M., Lawrence, J. R., Robarts, R. D., Caldwell, S. J. \& Caldwell D. E. (1994). Multicellular organization in a degradative biofilm community. Appl Environ Microbiol 60, 434-446.

Received 23 February 1999; revised 20 May 1999; accepted 4 June 1999. 